\title{
Formação dos Profissionais de Saúde na Comunicação de Más Notícias em Cuidados Paliativos Oncológicos
}

Health Care Professionals' Training in Communicating Bad News in Oncologic Palliative Care

\author{
Formación de Profesionales de la Salud en la Comunicación de Malas Noticias \\ en los Cuidados Paliativos Oncológica
}

\section{Barbara Rafaela Bastos ; Ana Carolina Galvão da Fonseca²; Adrya Karolinne da Silva Pereira ${ }^{3}$; Lorrany de Cássia de Souza e Silva}

\section{INTRODUÇÃO}

A medicina tem passado por diversas modificaçóes ao longo do tempo. É notável os avanços na prática médica, sobretudo nas tecnologias dos procedimentos invasivos e na prevenção/tratamento de doenças infectocontagiosas, esse fato tem originado melhorias relevantes na saúde. Em consequência disso, tornaram-se cada vez mais raros os casos de morte por alguns tipos de doença como, por exemplo, varíola e tuberculose ${ }^{1}$.

Fatores como a reduçáo da taxa de natalidade e mortalidade por doenças infectocontagiosas, o aumento das medidas preventivas em saúde e as práticas de vida saudável têm contribuído para o aumento da expectativa de vida populacional; em consequência disso, é maior a exposiçáo a fatores de risco, levando ao aumento da incidência de doenças crônico-degenerativas, especialmente as cardiovasculares e câncer ${ }^{2}$.

Dados do Instituto Brasileiro de Geografia e Estatística (IBGE) mostram que a expectativa de vida dos brasileiros aumentou em quase 30 anos de 1940 para $2014^{3}$. Esse fato, juntamente com o incremento das novas técnicas de diagnóstico, rastreamento e registro dos casos, como, por exemplo, os registros de câncer de bases populacionais e hospitalares, contribui para que mais pessoas sejam diagnosticadas com câncer ${ }^{4}$. No Brasil, as estimativas, para o biênio 2016-2017, apontam a ocorrência de aproximadamente 596.070 novos casos de câncer ${ }^{4}$.
Se por um lado, os avanços da medicina junto às condiçôes de vida e saúde têm proporcionado uma melhoria na qualidade de vida das pessoas, principalmente nas sociedades desenvolvidas; por outro, a questão do aumento da expectativa de vida náo pode ser confundido com prolongamento muitas vezes desnecessário e tratamentos injustificáveis, com a obstinação terapêutica a qualquer custo 5 .

Conceitua-se cuidados paliativos "como uma abordagem que visa a melhorar a qualidade de vida dos doentes, e das suas famílias, que enfrentam problemas decorrentes de uma doença incurável e com prognóstico limitado ${ }^{6}$. É importante frisar que a essência do cuidado paliativo só é efetiva se houver envolvimento da equipe multidisciplinar e uma comunicação efetiva, proporcionando uma assistência harmônica e convergente ao indivíduo sem possibilidades de cura e a sua família ${ }^{7,8}$.

A comunicação é um processo essencial nas relaçóes humanas, e é imprescindível para que se possa obter uma assistência de qualidade, além de estar voltada para o processo de humanização. A prática da comunicação é a medida terapêutica, comprovadamente eficaz, para os pacientes que carecem desses cuidados, sobretudo, os que se encontram em fase final de vida ${ }^{7,8}$.

A maneira de como comunicar uma má notícia pode ter um grande impacto no modo como a pessoa doente irá viver o processo saúde/doença e seu respectivo tratamento. Se a comunicaçáo for feita de maneira

\footnotetext{
${ }^{1}$ Enfermeira. Residente em Oncologia: Cuidados Paliativos pela Universidade do Estado do Pará (UEPA). Belém (PA), Brasil. E-mail: barbararafaela1@gmail.com

${ }^{2}$ Psicóloga. Residente em Oncologia: Cuidados Paliativos pela UEPA. Belém (PA), Brasil. E-mail: adrya-karolinne@hotmail.com

${ }^{3}$ Enfermeira. Residente em Oncologia: Cuidados Paliativos pela UEPA. Belém (PA), Brasil. E-mail: carolina.galvaofonseca@gmail.com

${ }^{4}$ Assistente Social. Residente em Oncologia: Cuidados Paliativos pela UEPA. Belém (PA), Brasil. E-mail: Lorranysouza_18@hotmail.com

Endereço para correspondência: Barbara Rafaela Bastos. Travessa Timbó, 1.839-Marco. Belém (PA), Brasil. CEP: 66095-128. E-mail: barbararafaela1@gmail.com
} 
inadequada, pode desencadear equívocos, sofrimento prolongado e ressentimento; se for feita adequadamente, pode gerar compreensão, aceitação e ajustamento ${ }^{8,9}$. Todos os profissionais de saúde precisam entender o processo de morte, e desenvolver principalmente a capacidade de se comunicar e "estar ao lado" quando a morte for inevitável ${ }^{10}$.

Visto que a comunicação é uma ferramenta essencial para essa modalidade de cuidado, como vem sendo aplicada à comunicação de más notícias em cuidados paliativos nos serviços de saúde? Os profissionais da área estariam preparados para estabelecer uma comunicaçáo adequada? As instituiçóes de ensino estariam fornecendo a formação necessária para efetivar o processo de comunicação nos cuidados paliativos?

\section{COMUNICAÇÃO DE MÁS NOTíCIAS EM CUIDADOS PALIATIVOS NOS SERVIÇOS DE SAÚDE}

$\mathrm{Na}$ prática dos cuidados de saúde, principalmente as voltadas para o processo de comunicação, são várias as questóes éticas que se apresentam aos profissionais de saúde. No domínio da relação entre profissionais de saúde e pacientes em processo de finitude, a comunicação de más notícias continua a ser uma das situaçôes mais delicadas, difíceis e complexas, quer pela gravidade das notícias, quer pelas indagaçôes que ainda existem em torno de quem, como, quando e o que comunicar à pessoa doente e sua família ${ }^{11}$. Por isso, levando em consideração a prática do cuidado paliativo, consideramos que a comunicação de más noticiais precisa ser aplicada e treinada por todos os profissionais envolvidos nesse contexto, pois sabemos que a maneira como a notícia é transmitida é um fator crucial para o enfrentamento de doenças que ameaçam a continuidade da vida.

Um projeto realizado pelo Ministério da Saúde, por intermédio do Instituto Nacional de Câncer José Alencar Gomes da Silva (INCA), e a Sociedade Beneficente Israelita Brasileira Albert Einstein, teve como tema central as dificuldades de comunicação de más notícias na prática clínica dos profissionais da saúde. Esse projeto foi descrito em um livro lançado em 2010 pelo INCA, sendo resultado de um laboratório de práticas com a intenção de beneficiar diretamente os profissionais envolvidos nessa atuação. Esse tipo de prática torna-se um valioso recurso para os profissionais da saúde, pois proporciona um espaço para a troca de experiências relacionadas às dificuldades enfrentadas no dia a dia ao lidar com comunicação de más notícias ${ }^{12}$.

Para que o repasse de más notícias seja adequado, os profissionais atuantes em cuidados paliativos precisam, além de aprender a se comunicar, reconhecer que o doente sempre tem alguma coisa a ensinar a toda equipe.
Quando se enxerga a possibilidade de se comunicar algo a alguém, deve-se estar preparado para responder aos questionamentos que forem apresentados de maneira segura e equilibrada. Se a equipe multidisciplinar estiver treinada para a comunicação de más notícias, isso será o início da terapêutica paliativa, proporcionando transparência na comunicação e estabelecendo vínculo de confiança com a equipe.

Vale ressaltar que a comunicação não verbal; ou seja, expressôes que não utilizam palavras, é também um componente essencial em todo esse processo, sendo que, muitas vezes, pode ser dedicada pouca atençáo por parte dos profissionais de saúde. A comunicação não verbal vai até mais além, pois permite a percepção e compreensão de sentimentos, dúvidas e angústias do paciente por meio de olhares e linguagem simbólica, típicos de quem está vivenciando a fase em que a cura náo é objetivo do tratamento ${ }^{13}$.

Destacamos a importância da comunicação de más notícias, pois implica diretamente na atitude do paciente, por isso, é indispensável e importante que o profissional estabeleça esse vínculo e esclareça todas as dúvidas em relação à assistência prestada proporcionando-lhe autonomia, elucidação e qualidade de vida. Além disso, precisa-se estar atento às tentativas de pacientes ao final da vida em descrever o que estâo vivendo, porque as informaçóes podem se perder se mal interpretadas ou ignoradas.

\section{CUIDAdos Paliativos E COMUNICAÇÃO Na FORMAÇão dOS PROFISSIONAIS DE SAÚDE}

Aprender a lidar com as perdas num contexto amplo é um desafio que poucos se propóem enfrentar, e muito menos conduzir. Ajudar indivíduos com doenças avançadas, sem possibilidades de cura, e ainda prestar apoio aos seus familiares em um dos momentos mais cruciais de suas vidas são atividades de extrema importância na atenção à saúde ${ }^{14}$.

Sendo assim, a falta de preparo dos profissionais para a comunicação e o suporte emocional aos pacientes geram silenciamentos, falsas promessas de cura ou comunicaçóes abruptas de prognósticos adversos, com sérios prejuízos à relação terapêutica. Percebemos que, embora seja importantíssimo saber se comunicar, ainda é escassa a inclusão do tema no ensino superior em saúde e nos programas de educaçáo continuada de muitas instituiçóes hospitalares.

Diante da falta de preparo, os profissionais de saúde necessitam constantemente aprimorar suas habilidades de comunicação, para saber o que, como e quando falar, assim como o momento de calar-se e ouvir paciente e familiares, mostrando-se solidário à dor do outro.

Estudo realizado em instituiçóes hospitalares de São Paulo sobre estratégias de comunicação utilizadas por profissionais de saúde na atenção a pacientes sob cuidados paliativos mostrou que todos os 303 profissionais estudados valorizavam imensamente a comunicação 
interpessoal no contexto da terminalidade. Contudo, o estudo evidenciou escasso conhecimento de estratégias de comunicação para a interação com pacientes sob cuidados paliativos: a maioria dos sujeitos $(57,7 \%)$ não foi capaz de citar ao menos uma estratégia adequada de comunicação verbal e apenas 15,2\% mencionaram sinais ou estratégias solicitadas. Isso pode sinalizar deficiência no processo de formação dos profissionais de saúde perante a comunicação de más notícias ${ }^{14}$.

\section{PAPEL DAS INSTITUIÇÕES DE ENSINO NA FORMAÇÃO DO PROCESSO DE COMUNICAÇÃO NOS CUIDADOS PALIATIVOS}

Alguns motivos que justificam a necessidade e a urgência da inclusão dos cuidados paliativos, juntamente com o manejo da comunicação de más notícias na grade curricular de formação dos profissionais de saúde, são o crescimento da expectativa de vida e o consequente aumento na ocorrência de doenças crônicas, como o câncer, que, por muitas vezes, se tornam uma ameaça à continuidade da vida ${ }^{15,16}$.

Deve-se dar maior ênfase nas escolas de ensino superior, pois, como são considerados espaços privilegiados para a construção de identidades sociais e profissionais, vêm se caracterizando por apresentar inúmeras limitaçóes, sobretudo quanto à formação no contexto paliativo e demandas psicossociais. As competências, habilidades e os conhecimentos adquiridos na qualificação profissional são insatisfatórios para atender às necessidades de assistência e de compreensão da subjetividade do indivíduo que lida com as perdas de autonomia e de esperança ${ }^{15,16}$.

Estudo sobre os cuidados paliativos, realizado em alunos de licenciatura em enfermagem, com o objetivo de identificar os conhecimentos sobre o tema, mostrou os seguintes resultados: $40,4 \%$ dos estudantes apresentam conhecimentos de nível insuficiente; 29,1\% suficiente; e $30,5 \%$ de nível bom ${ }^{16}$. Os dados revelam déficit de conhecimento dos alunos, mostrando que as instituiçóes de ensino devem capacitar seus alunos para uma comunicação eficaz diante da necessidade de fornecer más notícias.

Sabemos que, no mundo competitivo em que vivemos, a procura por profissionais tecnicamente capazes é cada vez mais intensa, isso faz com que o profissional procure aprimorar seu saber técnico-cientifico, entretanto, deixando de lado as habilidades emocionais e sociais, importantes nas relações humanas. É evidente a deficiência na formação dos profissionais de saúde de habilidades relacionadas aos cuidados paliativos, sendo as instituiçôes de ensino espaços estratégicos para difundir essa modalidade de cuidado.

Todos os profissionais de saúde e funcionários de instituições hospitalares, de forma geral, devem ser capazes de fornecer cuidados paliativos adequados e, portanto, precisam ser treinados para fornecer o mais elevado padrão de cuidados. Tudo isso com o objetivo de atender às necessidades e aos desafios dos pacientes e de seus familiares, independentemente do seu prognóstico ${ }^{17}$.

Sabe-se que, no Brasil, existem vários desafios a serem vencidos e, entre eles, está a possível deficiência na educação de profissionais de saúde no que diz respeito à terminalidade. Para superar essa deficiência, é preciso incluir o tema nas instituiçóes de ensino, assim como instituir ou ampliar programas de educação continuada que promovam a mudança de mentalidade dos profissionais para que possam conduzir adequadamente situaçôes relacionadas à terminalidade e à comunicação de más notícias.

\section{CONCLUSÃO}

Tendo em vista os aspectos ressaltados, consideramos que, na prática do cuidado paliativo oncológico, a comunicação, embora seja considerada uma ferramenta essencial na vida humana, ainda se faz complexa e deficiente.

Apesar do aumento do número de doenças crônico-degenerativas e do aumento da valorização da comunicação interpessoal no contexto da terminalidade, os profissionais de saúde ainda encontram dificuldades em desenvolver habilidades e conhecimentos necessários na comunicação de más notícias. Acredita-se que a formação dos profissionais de saúde deve incluir a comunicação de más notícias tanto na graduação quanto na educação continuada de instituições hospitalares.

Sugerimos que esses temas sejam inseridos como disciplina fundamental durante a graduação, pós-graduação, assim como no processo de educação continuada dentro das instituiçôes de saúde.

\section{CONTRIBUIÇÕES}

Todas as autoras participaram igualmente em todas as etapas de elaboração do artigo.

Declaraçáo de Conflito de Interesses: Nada a Declarar.

\section{REFERÊNCIAS}

1. Machado KDG, Pessini L, Hossne WS. A formação em cuidados paliativos da equipe que atua em unidade de terapia intensiva: um olhar da bioética. Bioethikos. 2007;1(1):34-42.

2. Carboni RM, Reppetto MA. Uma reflexão sobre a assistência à saúde do idoso no Brasil. Rev Eletrônica Enferm. 2007;9(1):251-60. 
3. Instituto Brasileiro de Geografia e Estatística. Tábua completa de mortalidade para o Brasil - 2014: breve análise da evolução da mortalidade no Brasil. Rio de Janeiro: IBGE; 2015.

4. Instituto Nacional de Câncer José Alencar Gomes da Silva. Estimativa 2016: Incidência de Câncer no Brasil. Rio de Janeiro: Inca; 2015.

5. Santos CKC, Andrade CG, Costa ICP, Lopes MEL, Silva CEG, Santos KFO. Comunicação em cuidados paliativos: revisáo integrativa da literatura. Rev Bras Ciênc Saúde. 2014;18(1):63-72.

6. World Health Organization. Cancer: WHO definition of palliative care [Internet]. Geneva: WHO; [acesso em 2017 jan. 27]. Disponível em: http://www.who.int/ cancer/palliative/definition/en.

7. Pontes AC, Leitão IMTA, Ramos IC. Comunicação terapêutica em Enfermagem: instrumento essencial do cuidado. Rev Bras Enferm. 2008;61(3):312-18.

8. Gonçalves ACR. Comunicação de más notícias a pessoas com doença oncológica: a necessidade de implementar a bioética na relação - um estudo exploratório [dissertação]. Lisboa: Universidade de Lisboa; 2013.

9. Araújo MMT, Silva MJP. A comunicação com o paciente em cuidados paliativos: valorizando a alegria e o otimismo. Rev Esc Enferm USP. 2007; 41(4):668-74.
10. Machado KDG, Pessini L, Hossne WS. A formação em cuidados paliativos da equipe que atua em unidade de terapia intensiva: um olhar da bioética. Bioethikos. 2007;1(1):34-42.

11. Fallowfield L, Jenkins V. Communicating sad, bad, and difficult news in medicine. Lancet. 2004;363(9405):312-9.

12. Instituto Nacional de Câncer (BR). Comunicação de notícias difíceis: compartilhando desafios na atenção à saúde. Rio de Janeiro: Inca; 2010.

13. Pessini L, Bertachini L. Humanização e cuidados paliativos. 3. ed. Sáo Paulo: Loyola; 2004.

14. Araújo MMT, Silva MJP. Estratégias de comunicação utilizadas por profissionais de saúde na atenção à pacientes sob cuidados paliativos. Rev Esc Enferm USP. 2012;46(3):626-32.

15. Gamondi C, Larkin P, Payne S. Competências Centrais em Cuidados Paliativos: Um Guia Orientador da EAPC sobre Educação em cuidados paliativos - parte 1 . European Journal of Palliative Care. 2013;20(2):140-45.

16. Galvão A, Lopes $\mathrm{S}$, Certo $\mathrm{AB}$. Cuidados paliativos em contexto de formação de licenciatura em enfermagem. In: ICHI 2014: Livro de resumos do Congresso Internacional de Informação em Saúde; 2014, Porto. Porto: Escola Superior de Enfermagem do Porto; 2014. p. 24.

17. Costa AP, Poles K, Silva AE. Formação em cuidados paliativos: experiência de alunos de medicina e enfermagem. 2016;20(59):1041-52. 